\title{
MASTER OT J172758.09+380021.5: a peculiar ER UMa-type dwarf nova, probably a missed nova in the recent past
}

\author{
E. Pavlenko ${ }^{1}$, T. Kato ${ }^{2}$, K. Antonyuk ${ }^{1}$, N.Pit ${ }^{1}$, L. Keir ${ }^{3}$, \\ S. Udovichenko ${ }^{3}$, P. Dubovský ${ }^{4}$, A. Sosnovskij ${ }^{1}$, O. Antonyuk ${ }^{1}$, \\ V.Shimansky ${ }^{5,6}$, M. Gabdeev ${ }^{5,6,7}$, F. Rakhmatullaeva ${ }^{8}$, \\ G. Kokhirova ${ }^{8}$, S. Belan ${ }^{1}$, A. Simon ${ }^{9}$, A. Baklanov ${ }^{1}$, \\ N. Kojiguchi ${ }^{2}$ and V. Godunova ${ }^{10}$ \\ 1 Federal State Budget Scientific Institution "Crimean Astrophysical \\ Observatory of RAS", Nauchny, 298409, Republic of Crimea, (E-mail: \\ eppavlenko@gmail.com) \\ 2 Department of Astronomy, Kyoto University, Kyoto 606-8502, Japan \\ 3 Astronomical Observatory, I. I. Mechnikov Odessa National University, \\ Odessa oblast, Ukraine \\ 4 Vihorlat Observatory, Mierova 4, 06601 Humenne, Slovakia \\ 5 Kazan (Volga region) Federal University, Kazan, 420008, \\ Kremlyovskaya 18, Russia \\ 6 Special Astrophysical Observatory, 369167, Nizhnij Arkhyz, \\ Karachai-Cherkessian Republic, Russia \\ 7 Tatarstan Academy of Science Institute of Advanced Studies, 4221, \\ Levobulachnaya St. 36 A, Kazan, Tatarstan republic, Russia \\ 8 Institute of Astrophysics of the Academy of Sciences of the Republic of \\ Tajikistan, Bukhoro 22, Dushanbe, 734042, Tajikistan \\ 9 Astronomy and Space Physics Department, Taras Shevshenko National \\ University of Kyiv, Volodymyrska str. 60, Kyiv, 01601, Ukraine \\ 10 ICAMER Oservatory of NAS of Ukraine, 27 Acad. Zabolotnogo str., \\ 03143 Kyiv, Ukraine
}

Received: November 5, 2020; Accepted: February 25, 2021

\begin{abstract}
A CCD photometry of the dwarf nova MASTER OT J172758.09 +380021.5 was carried out in 2019 during 134 nights. Observations covered three superoutbursts, five normal outbursts and quiescence between them. The available ASASSN and ZTF data for 2014 - 2020 were also examined. Spectral observations were done in 2020 when the object was in quiescence. Spectra and photometry revealed that the star is an H-rich active ER UMa-type dwarf nova with a highly variable supercycle (time interval between two successive superoutbursts) of $\sim 50-100 \mathrm{~d}$ that implies a high and variable mass-transfer rate. MASTER OT J172758.09+380021.5 demonstrated peculiar behaviour: short-lasted superoutbursts (a week); a slow superoutburst decline and cases
\end{abstract}


of rebrightenings; low frequency (from none to a few) of the normal outbursts during the supercycle. In 2019 a mean period of positive superhumps was found to be $0.05829 \mathrm{~d}$ during the superoutbursts. Late superhumps with a mean period of $0.057915 \mathrm{~d}$ which lasted about $\sim 20 \mathrm{~d}$ after the end of superoutburst and were replaced by an orbital period of $0.057026 \mathrm{~d}$ or its orbital-negative superhump beat period were detected. An absence of eclipse in the orbital light curve and its moderate amplitude are consistent with the orbital inclination of about $40^{\circ}$ found from spectroscopy. The blue peaks of the $V$ - Ic and $B-R c$ colour indices of superhumps during the superoutburst coincided with minima of the light curves, while $B-R c$ of the late superhumps coincided with a rising branch of the light curves. We found that a low mass ratio $q=0.08$ could explain most of the peculiarities of MASTER OT J172758.09+380021.5. The mass-transfer rate should be accordingly higher than what is expected from gravitational radiation only, this assumes the object is in a post-nova state and underwent a nova eruption relatively recently - hundreds of years ago. This object would provide probably the first observational evidence that a nova eruption can occur even in CVs near the period minimum.

Key words: stars: MASTER OT J172758.09+380021.5 - dwarf novae - activity

\section{Introduction}

Cataclysmic variables (CVs) are close binary systems in which a white dwarf (WD) accretes matter from a late-type donor star that fills its Roche lobe. Accretion is either disc-like or pole-on if a WD is non-magnetic or magnetic one, respectively. The orbital periods of CVs are limited by a rather blurry border of about 12 hours and by a strong period minimum at $78 \mathrm{~min}$ (Hellier, 2001). There is a so-called orbital period gap between 2.15 and 3.18 hours (Knigge, 2006) with a significant deficit of non-magnetic CVs. Non-magnetic CVs in the period gap and below it up to the period minimum are the SU UMa-type stars (Warner, 1995). These binaries are the H-rich stars approaching during their evolution to the period minimum or passed it ("period bouncers"), further evolution of period bouncers takes place with an increase of the orbital period. 56 system are known at present which have shorter periods than the period minimum. They are the He-rich AM CVn stars (Green et al., 2018) evolving with a decrease of the period. CVs on a way to AM CVn stars are called "EI Psc-type stars" (Kato et al., 2016a), they exhibit both $\mathrm{H}$ and He and are located near the period minimum. Another subgroup of SU UMa-stars, namely WZ-type stars, could also be found close to the period minimum (Kato, 2015). Transfer of matter from the late-type companion leads to thermal instability of the accretion disk and causes its outburst - a dwarf nova (DN) event (Osaki, 1996). SU UMa stars display two types of outbursts: the normal outbursts that last 2-5 days and brighter and longer superoutbursts lasting up to several weeks.

During the superoutbursts periodic brightness variations (positive superhumps) with periods of a few percent longer than the orbital period are present. 
These superhumps, as it was shown by Whitehurst (1988); Osaki (1989); Hirose \& Osaki (1990); Lubow (1991), are the consequence of tidal instability resulting from the 3:1 resonance in the accretion disc. This can only happen for the SU UMa-type stars with a mass ratio $q=M_{2} / M_{1} \leq 0.3$, where $M_{2}$ and $M_{1}$ are the masses of the donor and WD stars, respectively.

Kato et al. (2009) introduced three stages in the positive superhump evolution: Stage A has longer superhump periods and growth of superhump amplitudes; superhump periods during Stage B could systematically decrease/increase or be stable with a decrease of their amplitudes; Stage C has shorter superhump periods.

Some of the DNe display so-called late superhumps (Vogt, 1983). They have a similar period as positive superhumps, but have phases shifted by a half of this period. One explanation of this phenomenon is that they arise from a hot spot on an elliptical disc (Osaki, 1985).

Contrary to the positive superhumps, the negative superhumps have periods a few percents shorter than the orbital one. Negative superhumps mostly appear in quiescence independently on the mass ratio (Montgomery, 2010; Pavlenko et al., 2019) and probably are caused by nodal precession of a tilted accretion disk (Warner, 2003). Osaki \& Kato (2014) suggested that decrease of the frequency of the normal outbursts in some DNe resulting in a transition from a short (S) cycle to a long (L) one (designations of cycles, i.e., the interval between two successive outbursts, is taken from Smak (1985)) could be caused by a transition of a disc from coplanar with an orbital plane to a tilted to the orbital plane and the subsequent appearance of nodal precession and negative superhumps. This suggestion was confirmed for NY Her (Sosnovskij et al., 2017), V1504 Cyg (Osaki \& Kato, 2013) and V503 Cyg (Pavlenko et al., 2019).

WZ-type stars have in average a lowest mass transfer rate and rare outbursts that occur every several years - decades and actually are the superoutbursts with no normal outbursts with rare exceptions (Kato, 2015). One of the outstanding features of WZ Sge-type stars is rebrightenings, which are outbursts that appear after the plateau of the superoutburst during the superoutburst decline (Kato, 2015). WZ-type stars display a long-lasting approach to the quiescence. The superhumps during this stage (late-stage superhumps) could be observable, indicating that the disc in this stage is still thermally and tidally unstable.

About 25 years ago a special sub-group of SU UMa stars, ER UMa-type stars (Kato \& Kunjaya, 1995; Nogami et al., 1995), was distinguished among the DNe as having an extremely highest frequency of superoutbursts (every 20-50 days).

DN MASTER OT J172758.09+380021.5 (hereafter MASTER 1727) was discovered by Denisenko et al. (2014) in an outburst at $V=14^{m} .3$. Thorstensen et al. (2016) obtained spectrum in quiescence at $V \sim 18^{m}$.5. It showed very strong, relatively narrow single-peaked emission lines of $\mathrm{H}$. The emission-line velocities indicated an orbital period of 82.14(6) minutes. The authors predicted the superhumps detection in the nearest future. Indeed, superhumps were dis- 
covered in the 2019 superoutburst (see vsnet-alerts 22236 and 23335$)^{1}$, where the superhump period was reported to be 0.0565(1) d and 0.05803(1) d, respectively. K. Naoto previously suggested that this object might be the EI Psc-type candidate (vsnet-alert 23720). According to the available ASASSN (Shappee et al., 2014) light curve ${ }^{2}$ one could suggest a rather short supercycle (interval between two successive superoutbursts) of $\sim 50-60 \mathrm{~d}$ and unusually low frequency of normal outbursts between them within JD $2458415-2458650$.

In this paper, we present a spectral and photometric study of MASTER 1727 at different stages of its activity based on our observations in $2019-2020$ and on the ASASSN and $\mathrm{ZTF}^{3}$ (Masci et al., 2019) database in $2014-2019$.

\section{Observations and data reduction}

Spectral observations were carried out at the 6-m telescope of SAO RAS on 29 April 2020 using the SCORPIO reducer with a long slit mode. We used the volume holographic grating with 1200 lines per mm (VPHG1200G) and the 1 " slit. We obtained the spectra in the range $\lambda \lambda=3900-5700 \AA$ with the resolution of $5.5 \AA$. Five consecutive exposures with a total duration of 25 minutes were obtained. For the wavelength and flux calibration we used the HeNeAr lamp and a spectrophotometric standard. The observed data reduction was performed with standard methods under the $\mathrm{IDL}^{4}$ environment. Bright sky background, light clouds and seeing of stars about 2.5" provided the resulting $\mathrm{S} / \mathrm{N}$ ratio $\sim 40$

Photometric observations were carried out in 2019 during 134 nights (174 independed runs of observations) from June 25 to November 5 in the Crimean Astrophysical Observatory, Vihorlat Observatory, and in Observatories of Mayaki, Sanglokh and Terskol (see the log of observations given that is available in an electronic form). Most of the observations were done in white light (without any filter) but several observations close to the Johnson-Cousins $B, V, R c$ and $I c$ system were obtained. We used the comparison star 1280-0316078 of the USNO B1.0 catalogue. Depending on astroclimatic conditions (i.e. sky background, light absorption in the atmosphere and seeing), telescope and brightness of the object, exposure time varied and provided $S / N=20-200$. We used the MAXIM DL package for aperture photometry after standard data processing (i.e., debiasing, dark subtracting, flat-fielding).

For further analyses of the time series we used the Stellingwerf method implemented in the ISDA package (Pel't, 1980) and used the O-C analysis for the times of light curves maxima. For this and analysis of multicolour observations, we used the MCV program (Andronov \& Baklanov, 2004).

\footnotetext{
${ }^{1}$ https://groups.yahoo.com/neo/groups/cvnet-outburst/

${ }^{2}$ http://cv.asassn.astronomy.ohio-state.edu/

${ }^{3}$ https://lasair.roe.ac.uk/

${ }^{4}$ http://www.ittvis.com/idl
} 


\section{Results}

\subsection{Spectrum in the 2020 quiescence}

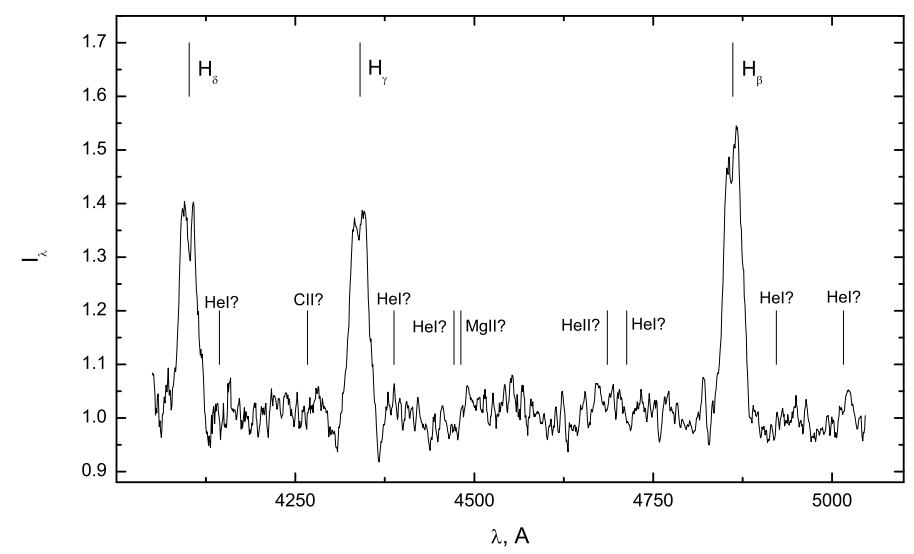

Figure 1. Spectrum of MASTER 1727 in the 2020 quiescence.

In the spectrum, double-peaked emission lines of the Balmer series with a $F W H M=23-27 \stackrel{\circ}{A}$ are observed (see Fig. 1). The lines of neutral and ionized He are not found with exception of a weak emission near $5015 \AA$, which probably is an artefact of sky lines subtraction. Besides of these lines, the CII and $\mathrm{Mg}$ II ions are also absent in the spectrum. Peak-to-peak distances in the HI $(\Delta \lambda=13.2-13.9 \AA)$ correspond to rotational velocity of the outer radius of the accretion $\operatorname{disc} V \sin (i)=410-450 \mathrm{~km} / \mathrm{s}$. Assuming the WD Roche-lobe radius $R_{L}=0.4 R_{\odot}$, its mass $M_{1}=0.75 M_{\odot}$ and the ratio of the outer radius of the accretion disk to the Roche-lobe size equal to 0.8 , one could estimate the orbital inclination $i=40^{\circ} \pm 2^{\circ}$.

According to the theoretical calculations of Paczynski (1977), the size of this radius is limited by the tidal effect of the secondary component and is about 0.8 $R_{L}^{1}$. To find the value of $R_{L}^{1}$ we applied the method for estimating the masses of the components of the close binaries developed by Borisov et al. (2017). The WD mass corresponds to $M_{1}=0.75 M_{\odot}$, the average mass of the primary component WD (Ritter \& Kolb, 2011). For a large set of mass ratios of the components $q=M_{2} / M_{1}$, we calculated the mass of the secondary component $M_{2}$ and the size of its Roche lobe according to the Eggleton formula (Eggleton, 1983):

$$
R_{L}^{2}=\frac{0.49 q^{2 / 3}}{0.6 q^{2 / 3}+\ln \left(1+q^{1 / 3}\right)}, \quad 0<q<\infty
$$

We estimated the radius of the secondary component $R_{2}$ for each value of its mass based on the results of evolutionary calculations of Baraffe et al. (2003) and 


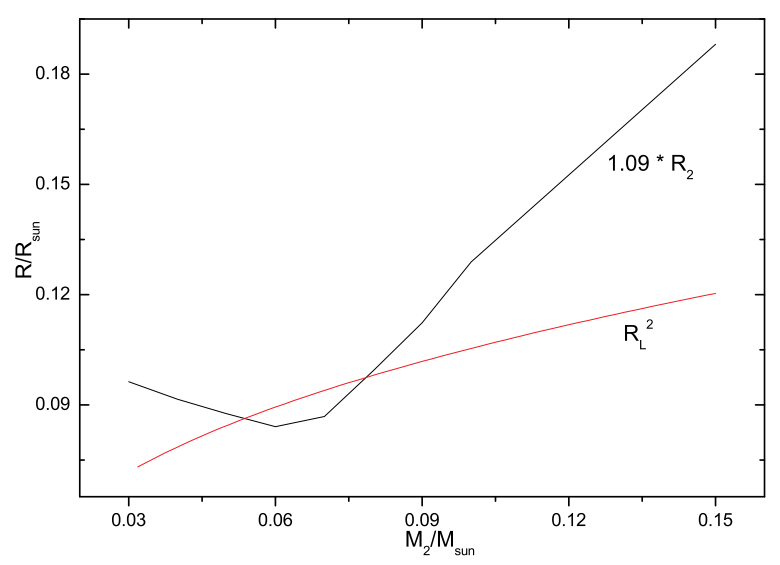

Figure 2. Dependences of the secondary star radius $R_{2}$ and its Roche-lobe radius $R_{L}^{2}$ on its mass $M_{2}$.

Girardi et al. (2000) for the low-mass dwarfs. The resulting dependences of $R_{2}$ and $R_{L}^{2}$ on $M_{2}$ were compared (see Fig. 2) taking into account the assumption that for systems with stable accretion the following condition is valid:

$$
R_{L}^{2}=1.05-1.09 \sim R_{2}
$$

For $M_{2}=0.070 M_{\odot} \pm 0.005 M_{\odot}$, ensuring the fulfillment of the condition, we calculated by the formula (Eggleton, 1983) Roche-lobe radius of the primary component $R_{L}^{1}=0.347 R_{\odot} \pm 0.030 R_{\odot}$ and the outer radius of the accretion disk $R_{A D}=0.28 R_{\odot} \pm 0.03 R_{\odot}$. Comparing the Keplerian velocity at this radius $V=690 \mathrm{~km} / \mathrm{s}$ with the observed values presented above we can find the angle of inclination of the orbit is $i=41^{\circ} \pm 5^{\circ}$.

\subsection{A long-term light curve}

In this subsection, we consider supercycles, cycles and morphology of superoutbursts based on our data obtained in 2019 and on the available ASASSN and ZTF data obtained in $2014-2019$.

\subsubsection{9 light curve}

The overall 2019 light curve of Master 1727 is shown in Fig. 3. It is constructed using our and part of ASASSN data. It covered three superoutbursts with the amplitude of $\sim 4^{m}$ and four normal outbursts. All superoutbursts displayed an unusually short duration of about one week. This is shorter than a range of 


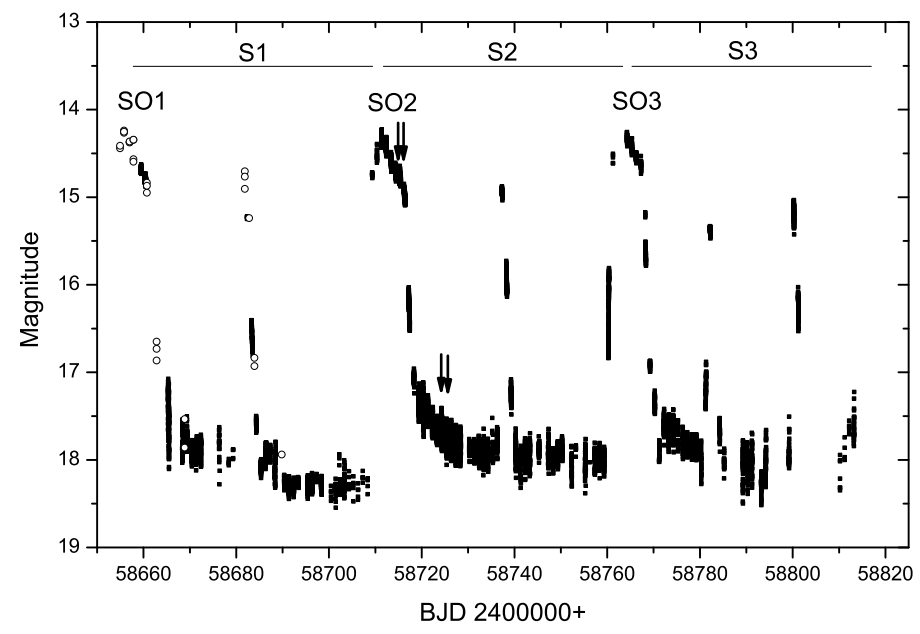

Figure 3. An overall 2019 light curve of MASTER 1727. SO1, SO2 and SO3 denote the number of superoutbursts while S1, S2 and S3 denote the number of supercycles. Our and ASASSN data are marked by black and open circles, respectively. Arrows indicate the time of colourimetric observations.

typical H-rich DNe (Warner, 1995) with exception of the 6-d duration in RZ LMi (Robertson et al., 1995), though there are a few He-rich AM CVn systems (Cannizzo \& Ramsay, 2019) with a duration of superoutbursts less than 10 days. The superoutburst plateau of MASTER $1727+38$ declined with a rate of $\sim 0.136$ $\mathrm{mag} / \mathrm{d}$. Then the superoutburst displayed rapid decay of $\sim 2.5 \mathrm{mag}$ in two days, subsequent slow return to quiescence that lasted up to the beginning of the next superoutburst, and a short supercycle of $52 \mathrm{~d}$. The normal outbursts lasted about two days. There was only one normal outburst just in the middle between superoutbursts, this activity repeated for two neighbour supercycles. However, the next supercycle included at least two normal outbursts. The appearance of outbursts during a slow approaching of superoutbursts to quiescence looks like rebrightenings often seen in the WZ Sge-type stars (Kato, 2015).

\subsubsection{ASASSN and ZTF 2014 - 2019 light curve}

It was possible to explore the outburst activity of MASTER 1727 on the $\sim 5.5$ year scale $(2014$ - 2019) using the available ASASSN and ZTF data (see Fig. 4). Contrary to our observations, we cannot immediately identify a type of all bright events (whether they are normal outbursts or superoutbursts) for this data. Moreover, in the case of the the superoutburst, maximum of brightness potentially could refer to any moment within the 7-day plateau. Probably all the data of $14^{m}-14.5^{m}$ refer to the superoutbursts. The impression from the visual inspection of the data is that the supercycle is not constant on a 5.5-year 


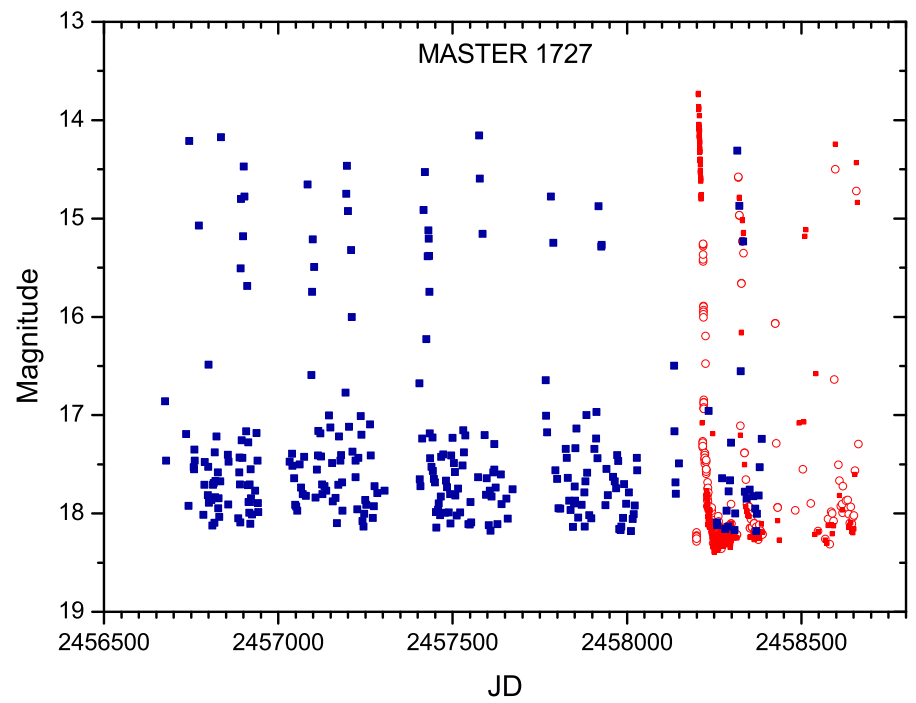

Figure 4. The 5.5-year light curve. ASASSN and ZTF data are shown by blue and red colours, respectively.

scale. Due to the short duration of the superoutburst, risk of missing it in a not very dense observation series makes it difficult to accurately determine the supercycle length. Nevertheless, denser observations (also taking into account our data) allow us to conclude that the supercycle could change in a range of 52 - 110 d during 1.5 years at least in 2018 - 2019. Note that no normal outbursts occurred during the 110-d supercycle. Of all the data on a 5.5-year scale, six events with a complex profile can be distinguished (Fig.5). The most striking among them are the fifth and sixth events. The fifth event obviously is the superoutburst with two rebrightenings. A plateau of this superoutburst lasted a $\sim$ week. The sixth event could be understood as a superoutburst lasting $6-9 \mathrm{~d}$ and the subsequent rebrightening with an unusually long $(\sim 6 \mathrm{~d})$ rising branch. The first - fourth events probably can also be related to superhumps with rebrightenings.

\subsection{Superhumps during 2019 SO2 superoutburst}

Due to the fact that the SO2 superoutburst plateau was closely covered by observations, we calculated its mean superhump period. A periodogram indicates the most significant period of $0.05803(4) \mathrm{d}$ among the one-day aliased peaks (see Fig. 6) that we attribute to the positive superhump period. Its mean the profile is asymmetric one with an amplitude of about $0^{m} .1$. Over a course of all superoutbursts and in more detail during the SO2 superoutburst (Fig.8), the original amplitudes of superhumps decrease from $0^{m} .15$ to $0^{m} .05$ This means that su- 

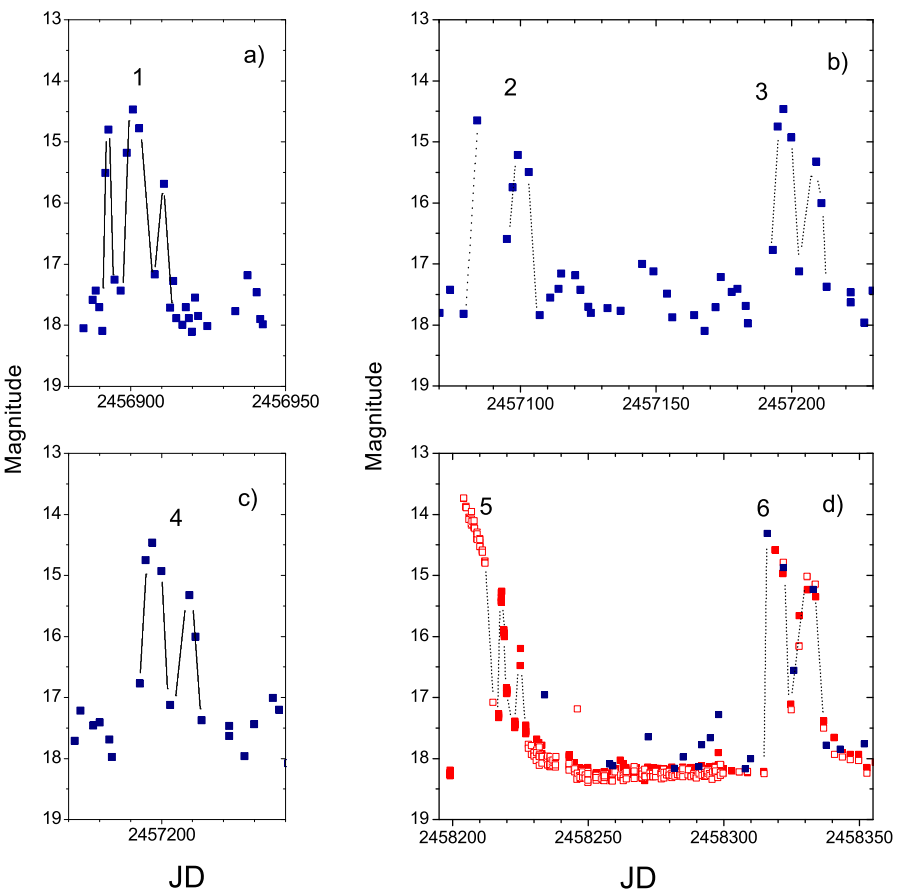

Figure 5. Fragments (a, b, c, d) of the long-term light curve displaying complex morphology of the outburst profile. The first event probably associates with a normal outburst + superoutburst + rebrightening, the second - fourth events and the sixth one with the superoutburst + rebrightening and the fifth event with the superoutburst and two rebrightenings. ASASSN and ZTF data are shown by blue and red colours, respectively.

perhumps were already fully grown since the superoutburst maxima (stage B) and stage A was probably too brief (a day or so) to be catched by us.

The periodic light variations with a period close to a superhump period continued over the quiescence between superoutbursts during every supercycle. The times of maxima for nightly light curves were defined wherever possible (see Tab. 1) and (O-C)s were calculated using the zero epoch of BJD 2458710.346 and the period of $0.0580294 \mathrm{~d}$. The O-C for the superhumps maxima of the SO2 superoutburst displayed a possibly slight increase of the mean period.

\subsection{Periodicity in quiescence}

Starting with BJD 2458717, which was a middle of the fast SO2 superoutburst decline, the (O-C)s displayed a clear jump by a half the period of positive superhumps. This event looked like classical late superhumps (Vogt, 1983; Kato 

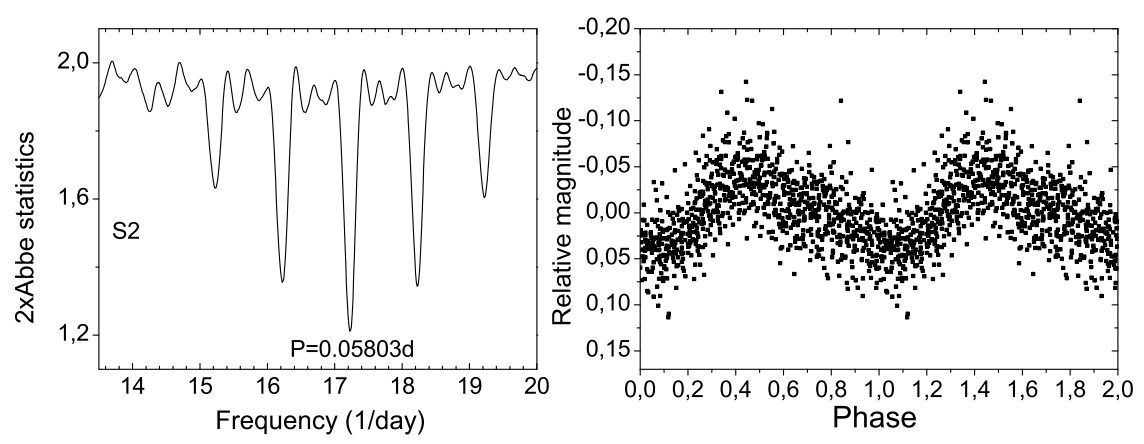

Figure 6. Left: a periodogram for the $\mathrm{S} 2$ superoutburst plateau in the vicinity of the superhump period. A corresponding zero epoch is 2458711.29888 . The most significant peak indicates the period of $0.05803(4) \mathrm{d}$. Right: data folded on this period.

et al., 2002; Uemura et al., 2002) that is shown in Fig.7 and Fig.8. Further (OC)s decreased up to the next superoutburst that means that the corresponding period was shorter than superhumps in the superoutburst. Simultaneously the mean amplitude of this periodicity grew from $0^{m} .05$ to $0^{m} .35$ up to the onset of the normal outburst. After that, the cycle-to-cycle amplitudes varied around the mean amplitude of $\sim 0^{m} .15$ up to the end of the supercycle.

It seems that the $\mathrm{O}-\mathrm{C}$ behaviour repeated from supercycle to supercycle. To study the nature of periods in quiescence we combined the O-C for all S1, S2 and S3 supercycles by choosing the zero-point as the onset of the corresponding superoutburst. Despite poorer statistics for S1 and S3 supercycles, the O-C behaviours are consistent with each other. We have identified three stages in the O-C behaviour: a stage of the superoutburst plateau Sa, quiescence Sb that is limited by the end of the superoutburst and the normal outburst, and quiescence Sc between the end of the normal outburst and the next superoutburst (see Fig. 9). Limited and uneven coverage of the superhump plateau does not enable us to define the evolution of superhumps during stage $B$. The mean period at this stage is $0.058029 \mathrm{~d}$. The stages $\mathrm{Sb}$ and $\mathrm{Sc}$ could be fitted by the linear trends which refer to two periods of $0.057938 \mathrm{~d}$ and $0.057611 \mathrm{~d}$, respectively. Alternatively, $\mathrm{Sb}+\mathrm{Sc}$ stages could be approximated by a parabola. The scattering around linear fits seems to be slightly less than around the parabola, so the version of two periods in quiescence is more reliable.

We performed a periodogram analysis for the data at $\mathrm{Sb}$ and $\mathrm{Sc}$ stages using data of the S2 supercycle only (Fig. 10). The periods at these stages were $0.057915(10) \mathrm{d}$ and $0.057026(10) \mathrm{d}$, respectively. One could see a good agreement of the $\mathrm{O}-\mathrm{C}$ and periodogram analyses for the period at the Sb stage. The mean period at the stage $\mathrm{Sb}$ is $0.00011 \mathrm{~d}$ less than the mean period at the Sa stage. We suggest that this is a period of late superhumps that lasted about $20 \mathrm{~d}$ up to 
Table 1. Times of nightly light curves maxima of the MASTER J1727 in 2019.

\begin{tabular}{|c|c|c|c|c|c|c|c|}
\hline $\begin{array}{l}\text { BJD } \\
2458000+\end{array}$ & Error & $\begin{array}{l}\text { BJD } \\
2458000+\end{array}$ & Error & $\begin{array}{l}\text { BJD } \\
2458000+\end{array}$ & Error & $\begin{array}{l}\text { BJD } \\
2458000+\end{array}$ & Error \\
\hline 659.457 & 0.003 & 715.212 & 0.003 & 722.318 & 0.004 & 733.324 & 0.003 \\
\hline 659.517 & 0.003 & 715.270 & 0.002 & 722.324 & 0.003 & 736.332 & 0.005 \\
\hline 660.333 & 0.004 & 715.330 & 0.005 & 722.325 & 0.002 & 736.343 & 0.004 \\
\hline 660.395 & 0.003 & 715.331 & 0.002 & 722.381 & 0.002 & 738.264 & 0.005 \\
\hline 660.456 & 0.002 & 715.332 & 0.003 & 722.441 & 0.003 & 743.298 & 0.006 \\
\hline 665.356 & 0.004 & 715.389 & 0.003 & 723.252 & 0.002 & 745.245 & 0.007 \\
\hline 665.419 & 0.003 & 715.445 & 0.004 & 723.311 & 0.003 & 747.293 & 0.005 \\
\hline 668.377 & 0.003 & 716.144 & 0.003 & 723.375 & 0.003 & 748.328 & 0.006 \\
\hline 668.432 & 0.003 & 716.202 & 0.003 & 724.298 & 0.002 & 749.230 & 0.004 \\
\hline 669.415 & 0.003 & 716.316 & 0.002 & 724.359 & 0.002 & 749.290 & 0.005 \\
\hline 670.400 & 0.003 & 716.317 & 0.003 & 724.414 & 0.002 & 749.325 & 0.006 \\
\hline 670.456 & 0.003 & 716.318 & 0.005 & 725.279 & 0.002 & 752.311 & 0.003 \\
\hline 671.380 & 0.008 & 716.375 & 0.003 & 725.279 & 0.003 & 755.317 & 0.004 \\
\hline 671.503 & 0.005 & 716.376 & 0.002 & 725.336 & 0.002 & 759.315 & 0.003 \\
\hline 672.400 & 0.003 & 716.435 & 0.003 & 725.390 & 0.004 & 760.297 & 0.005 \\
\hline 683.319 & 0.01 & 717.160 & 0.003 & 725.396 & 0.002 & 764.296 & 0.004 \\
\hline 683.445 & 0.008 & 717.225 & 0.004 & 726.319 & 0.003 & 765.213 & 0.003 \\
\hline 686.347 & 0.005 & 717.396 & 0.003 & 727.255 & 0.002 & 766.265 & 0.005 \\
\hline 686.410 & 0.007 & 718.317 & 0.003 & 727.310 & 0.008 & 770.243 & 0.005 \\
\hline 691.390 & 0.01 & 719.300 & 0.004 & 727.314 & 0.004 & 770.295 & 0.005 \\
\hline 710.346 & 0.002 & 719.301 & 0.003 & 727.368 & 0.008 & 772.288 & 0.004 \\
\hline 711.331 & 0.002 & 719.421 & 0.003 & 727.373 & 0.004 & 772.261 & 0.004 \\
\hline 712.311 & 0.002 & 720.292 & 0.004 & 728.293 & 0.004 & 773.263 & 0.004 \\
\hline 712.311 & 0.003 & 720.295 & 0.003 & 728.296 & 0.004 & 774.251 & 0.002 \\
\hline 712.370 & 0.005 & 720.356 & 0.005 & 728.347 & 0.004 & 776.276 & 0.005 \\
\hline 713.360 & 0.005 & 720.360 & 0.003 & 728.352 & 0.003 & 778.241 & 0.002 \\
\hline 713.412 & 0.003 & 720.361 & 0.003 & 730.253 & 0.003 & 779.232 & 0.002 \\
\hline 714.288 & 0.003 & 721.280 & 0.003 & 730.269 & 0.004 & 781.246 & 0.003 \\
\hline 714.342 & 0.005 & 721.281 & 0.002 & 731.309 & 0.003 & 782.185 & 0.003 \\
\hline 714.343 & 0.004 & 721.332 & 0.003 & 731.368 & 0.003 & 782.223 & 0.004 \\
\hline 714.398 & 0.002 & 721.334 & 0.003 & 731.368 & 0.003 & 791.258 & 0.005 \\
\hline 714.402 & 0.003 & 721.394 & 0.005 & 732.344 & 0.002 & 800.220 & 0.005 \\
\hline 714.460 & 0.003 & 722.268 & 0.002 & 732.400 & 0.003 & 801.192 & 0.005 \\
\hline
\end{tabular}

the start of a normal outburst. Note that IY UMa (Patterson et al., 2000) also displayed the period of late superhumps that was slightly shorter than during the superoutburst plateau. As for the Sc stage, the O-C also yields confirmation of the period obtained from the periodogram analysis but with less accuracy (probably because of an insufficient number of the $\mathrm{O}-\mathrm{C}$ data). We adopt the $0.057026(9) \mathrm{d}$ for the orbital period.

According to Hellier (2001) an expected fractional period deficit $\epsilon^{-}$of nega- 


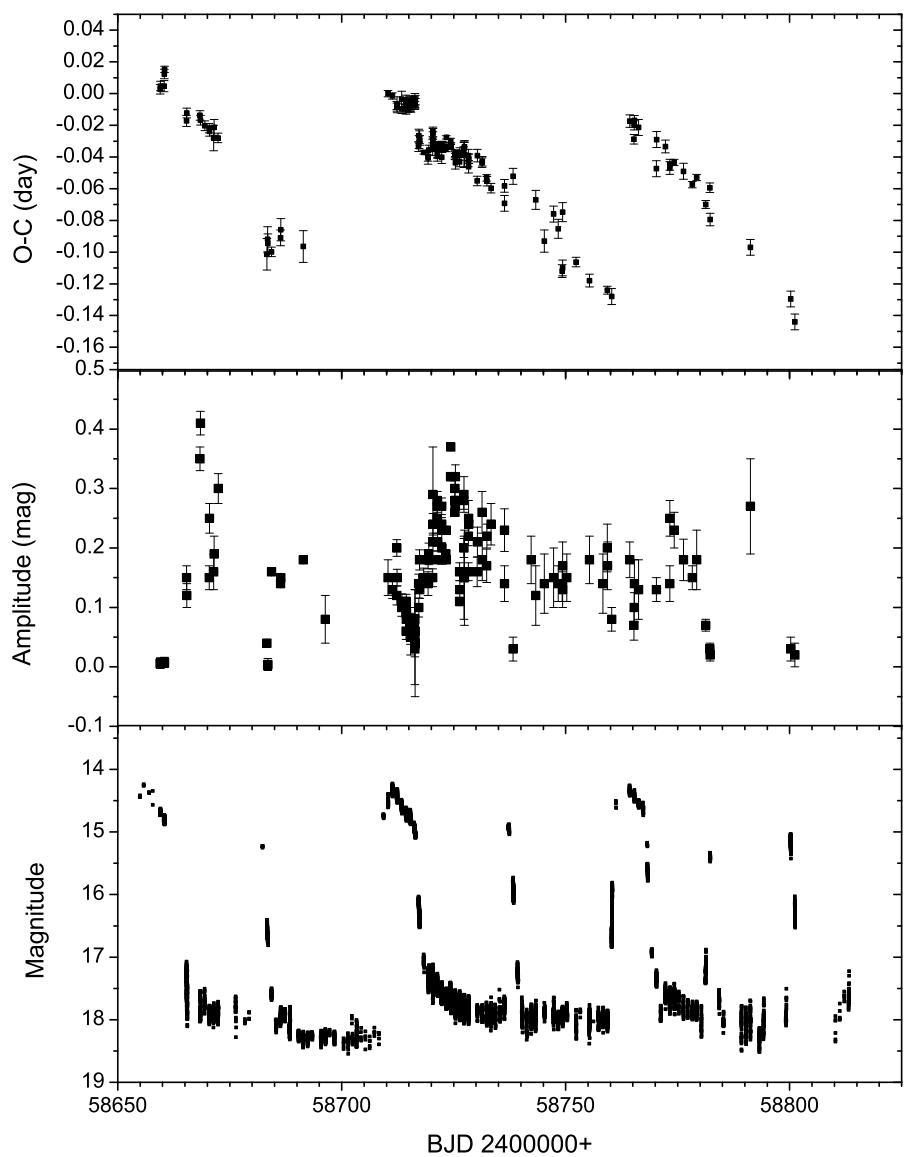

Figure 7. From top to bottom: O-C for maxima; amplitudes and an overall light curve during three S1, S2 and S3 supercycles.

tive superhumps is $\sim 0.5 \times \epsilon^{+}$(here $\epsilon^{+}$is a fractional period excess). In our case $\epsilon^{-}$should be $\sim-0.0087$ and the period of negative superhumps has to be about $0.0565 \mathrm{~d}$ (frequency $17.70 \mathrm{~d}^{-1}$ ). No prominent peak at this frequency for both the superoutburst and the quiescence was found. There is only a week signal at this frequency during the stage $\mathrm{C}$ that we cannot take into account due to its low significance. Instead, a period of $0.057026(9) \mathrm{d}$ within errors coincides with the orbital period $0.057042(42) \mathrm{d}$ that was obtained from spectroscopy (Thorstensen et al., 2016). The mean light curve does not contain the eclipse and has a relatively low amplitude (about $0^{m} .1$ ). This does not contradict the moderate inclination of the orbit $\left(i=40^{\circ} \pm 2^{\circ}\right)$ that we obtained from spectroscopy. 


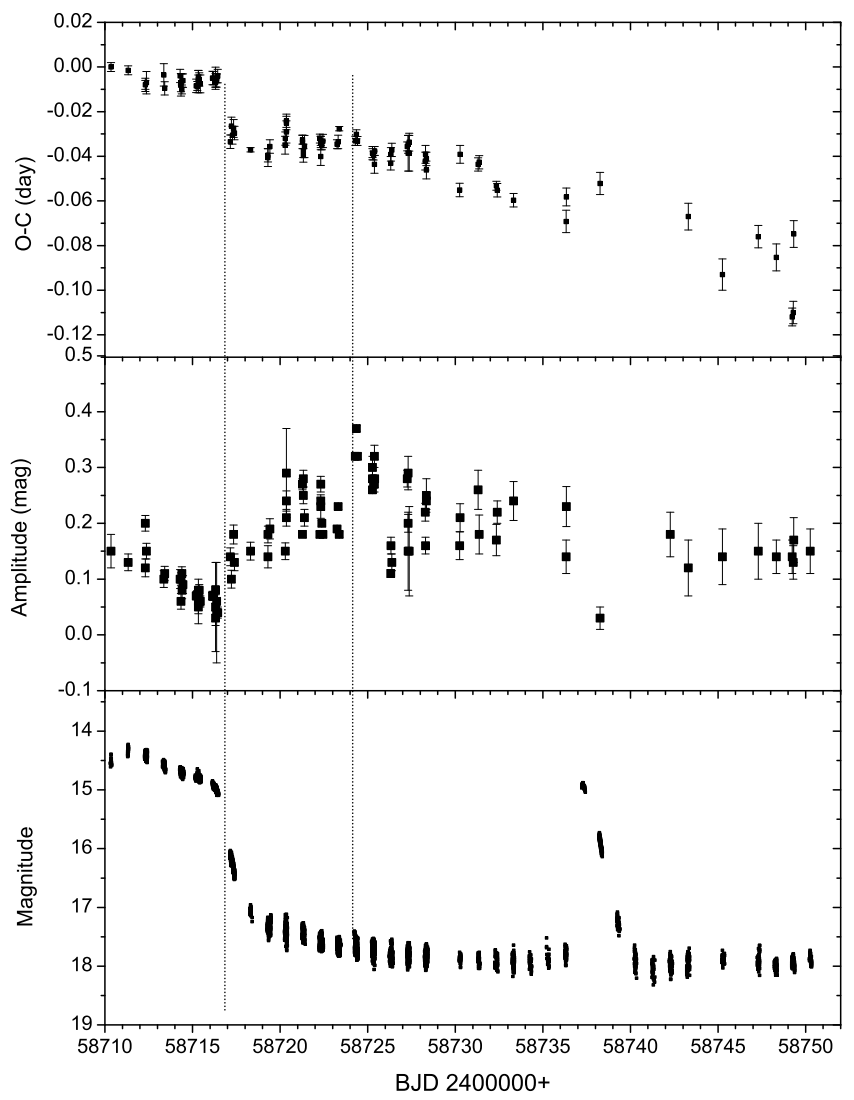

Figure 8. The same as in Fig. 7, but for the part of the S2 supercycle only.

Despite the insufficiently dense rows of the ASASSN and ZTF data for each night, a large number of nights in the quiescent state following the fifth superoutburst made it possible to search for periodicities in this quiescence. We have done this for data of a slow decline (JD 2458227 - 2458250) and quiescence (JD 2458253 - 2458315) separately. A result is presented in Fig.11.

A periodogram for the slow superoutburst decline revealed a most significant period of $0.057929(7)$ d. It coincides, within accuracy, with the period of late superhumps 0.057915(10) d found in 2019. Among the day-aliased peaks of periodogram for quiescent data one peak points to a period of $0.057797(3) \mathrm{d}$ that is close to but is slightly less then the period of the late superhumps. In the case of our observations in 2019, where the second half of the supercycle was presented by orbital variations, the ZTF periodogram does not show this period. Possibly a period of late superhumps acted in quiescence and was somewhat smaller than during a slow fading, or there was cross-contamination of the late 


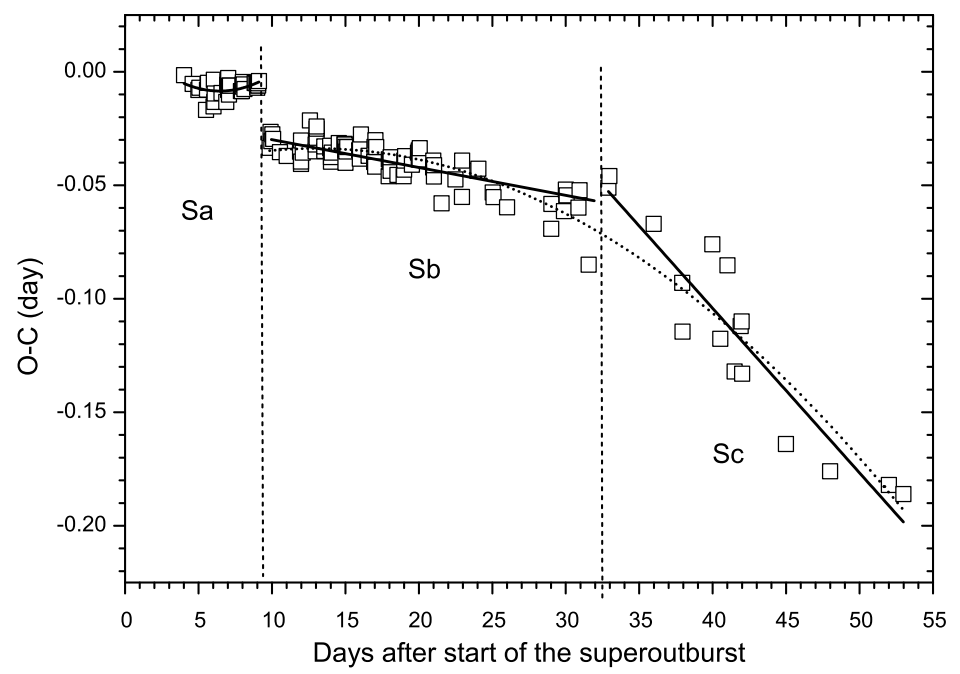

Figure 9. O-C combined for three S1, S2 and S3 supercycles. Sa, Sb and Sc mean three stages of combined supercycles: Sa means a plateau of the superoutburst, $\mathrm{Sb}$ and Sc are the parts of supposed late superhumps. The dashed curve is the best parabolic fit of (O-C)s behaviour at $S b+S c$ stages; solid lines are the best fits of $(\mathrm{O}-\mathrm{C}) \mathrm{s}$ at $\mathrm{Sb}$ and Sc stages separately.
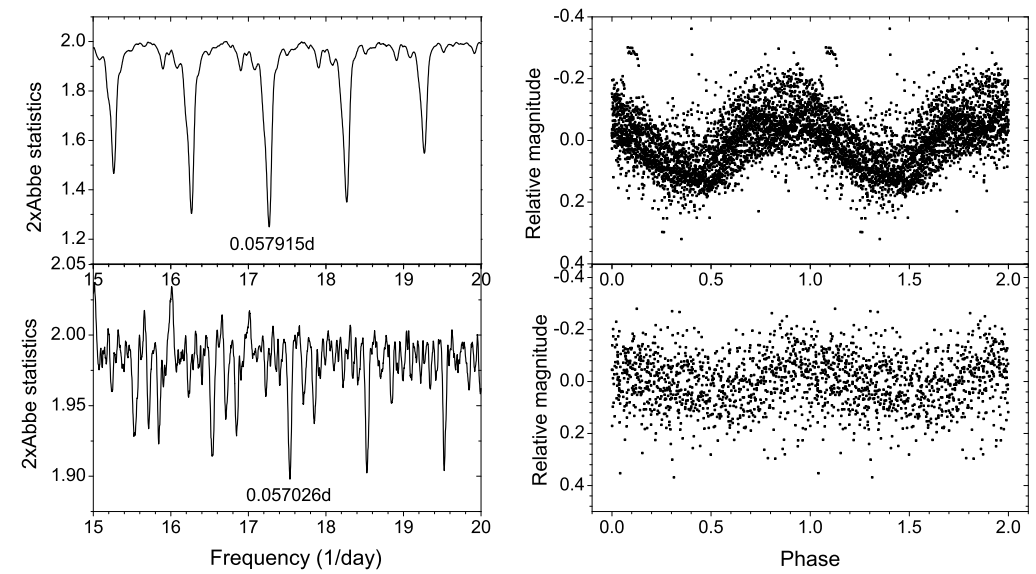

Figure 10. Left from top to bottom: periodograms for data in the S2 quiescence before the normal outburst and after it; Right from top to bottom: data folded with the most significant periods $0.057915 \mathrm{~d}$ and zero epoch $2458725.253 ; 0.057026 \mathrm{~d}$ and zero epoch 2458740.252 , respectively. 

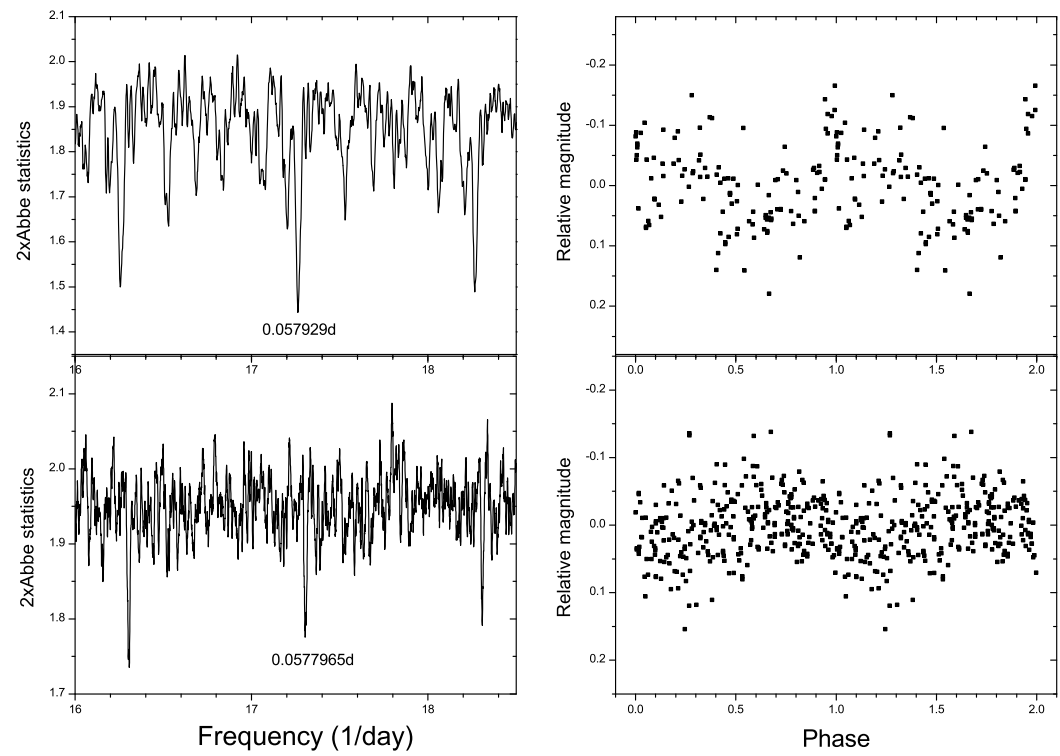

Figure 11. Left from top to bottom: a periodogram for data of a slow superoutburst 6 decline and the subsequent quiescence; right from top to bottom: data folded on the periods of $0.057929 \mathrm{~d}$ and $0.0577965 \mathrm{~d}$, respectively.

superhumps, orbital periodicity, potential negative superhumps or some of them (the negative superhumps could be good candidates to an additional periodicity in the quiescence taking into account that this supercycle does not contain normal outbursts, so we can consider it as an L-type supercycle). The negative fractional period excess is poorly defined on the empirical diagram "orbital period $-\epsilon^{-} "$ (Hellier, 2001) for the shortest orbital periods. The extrapolation of the data suggests an expected $\epsilon^{-}$in a region from -0.008 to -0.02 . In the case of the orbital frequency $F_{\text {orb }}=17.536$, a frequency of variations in quiescence $F_{q}=17.302$ could be a beat (side-band) frequency $F_{s b}=2 F_{\text {orb }}-F_{n}$ for $\epsilon^{-}=$ -0.013 and the frequency of negative superhump $F_{n}=17.767$.

\subsection{Color indices}

The multicolour $V, I c$ and $B, R c$ observations of superhumps were done for two nights of the $\mathrm{S} 2$ superoutburst plateau and those of $B, R c$ were done during the slow S2 superoutburst decline close to quiescence. As it follows from Fig. 12, colour indices are the phase-dependent ones and their dependence is different for different stages of outburst activity. During the S2 superoutburst, the bluest peak of the $V-I c$ and $B$-Rc coincided with a maximum of the light curve, while during a slow approach to quiescence it was slightly shifted and coincided with 
a rising branch of the light curve. The amplitudes of colour curves were $\sim$ $0^{m} .08$ during the superoutburst and $\sim 0^{m} .1-0^{m} .2$ close to quiescence. Similar behaviour of the superhumps colour indices was reported for several SU UMa stars (see a brief review by Pavlenko et al. (2020).

\subsection{Mass ratio from photometry}

With known orbital and positive superhumps periods, one could calculate the fractional period excess $\epsilon^{+}$at stage B of the superoutburst

$$
\epsilon^{+}=(\text {Psh }- \text { Porb }) / \text { Porb }
$$

where Psh and Porb are positive superhump and orbital periods, $q=M_{2} / M_{1}$, $M_{2}$ and $M_{1}$ are the masses of the donor and the primary components, respectively. We obtained $\epsilon^{+}=0.0173$ for the stage B.

With a known orbital period and $\epsilon$ we can estimate the mass ratio $q\left(\epsilon^{+}\right)$. According to Kato \& Osaki (2013), the real $q$ one could obtain with the period of superhumps that is detected at stage A of the superoutburst, which is unknown in our case. As we pointed in Section 5, stage A was not recorded in any of the superoutbursts: it is seen from Fig. 7 and Fig. 8 that the onset of the superoutburst is already accompanied with fully grown superhumps. The only thing that can be said is that a potential stage A lasted no more than a day. However, we could estimate expected $\epsilon^{*}$ at stage A using the relation of Kato \& Osaki (2013),

$$
\epsilon^{*}=0.012(2)+1.04(8) \epsilon^{+},
$$

where $\epsilon^{*}$ and $\epsilon^{+}$are the fractional period excesses at stage A and B, respectively. We found $\epsilon^{*}=0.030$. Then, according to Table 1 from Kato \& Osaki (2013), we obtain $q=0.081$ and assuming $M_{1}=0.75 M_{\odot}$, we have $M_{2}=0.061 M_{\odot}$.

This result is in a good agreement with the estimate for the mass described in Section 3 and places MASTER 1727 near a low-mass bound of the H-rich SU UMa stars (Fig. 13).

\section{Discussion: MASTER 1727 as a peculiar ER UMa-type star}

In the 90s the known supercycles of SU UMa-type stars were mainly between $\sim 100$ and $\sim 500$ days (Kato et al., 1999). A special group of four objects with supercycles of 19 - 44 d, called ER UMa-type stars (Nogami et al., 2003), stood out among them. At that time, no new SU UMa type-stars were known between these two groups. Over time, information began to appear about both new stars of the ER UMa-type and stars with supercycles less than 100 d ("active novae"). Moreover, as it turned out, ER UMa itself displayed a change of its supercycle (Zemko et al., 2013) in a range of 42-60 d. A modern histogram of the supercycles of SU UMa-stars distribution is presented in Fig. 14. This 

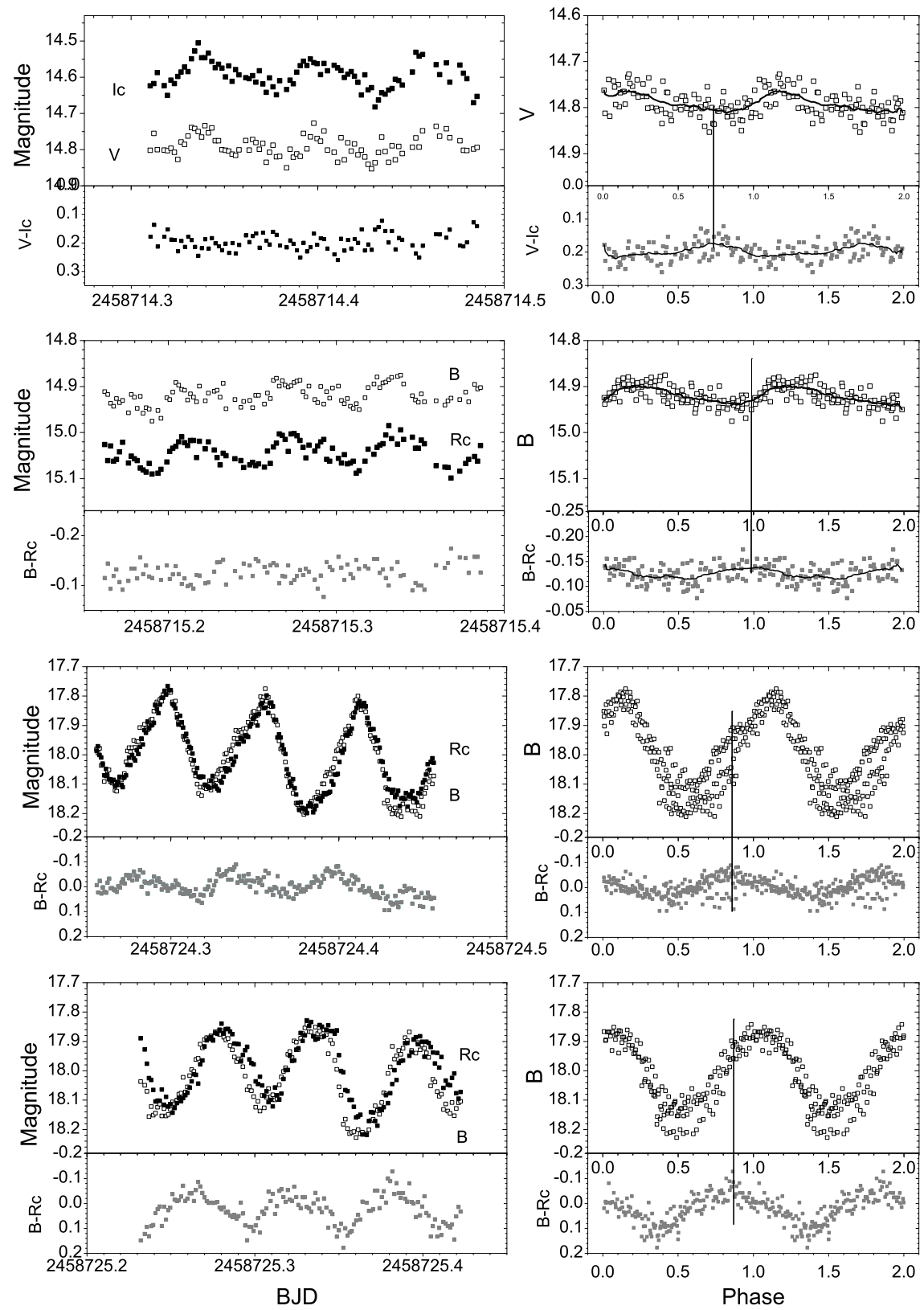

Figure 12. Left from top to bottom: original light and colour indices curves for the data of the S2 superoutburst plateau (BJD 2458714 and 2458715 ) and two consecutive nights close to quiescence (BJD 2458724 and 2458725). Right from top to bottom: folded light ( $V$ and $I c, B$ and $R c$ ) and instrumental colour indices ( $V$ - $I c$ and $B-R c$ ) on the $0.058029 \mathrm{~d}$ period with the zero epoch BJD 2458724.0. The dotted line is plotted through the $B-R c$ maximum. The smoothed line is drawn through the $V, V-I c$, and $B, B-R c$ light and colour indices curves, respectively. 


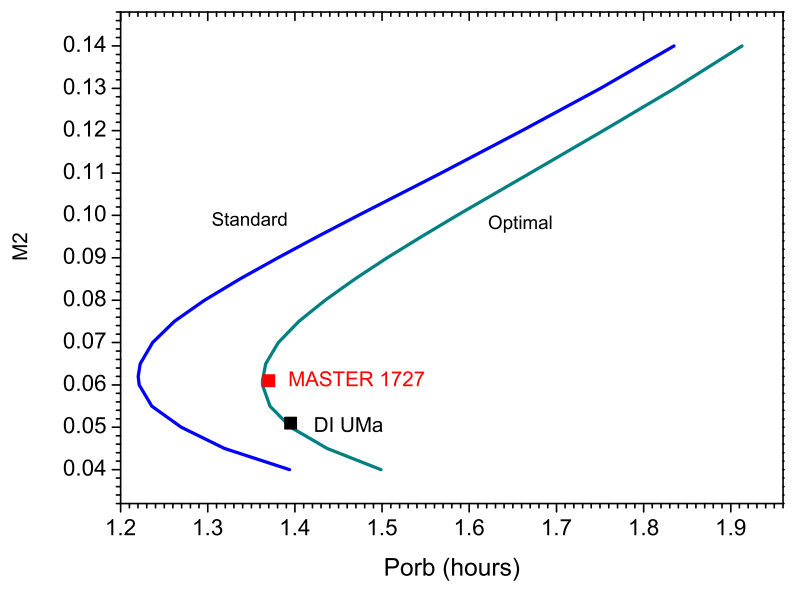

Figure 13. Dependence of the mass of the secondary $M_{2}$, expressed in units of the solar masses, on the orbital period. For DI UMa we used $P_{o r b}=0.054564 \mathrm{~d}$ (Fried et al., 1999) and re-estimated $q=0.068$ and $M_{2}=0.051 M_{\odot}$ obtained by the same method as for MASTER 1727. Tracks of standard and optional evolution according to Knigge et al. (2011) are marked by blue and dark cyan lines, respectively. The position of DI UMa and MASTER 1727 is shown.

distribution is a bimodal one. The largest number of supercycles falls on 250400 days (the primary maximum). A smaller, almost flat secondary maximum falls on the interval of 40-160 days and ER UMa-type stars no longer look like a separate group in this distribution.

A short 50-d supercycle length could define MASTER 1727 as an ER UMatype DN. Also, like other ER UMa-type stars, MASTER 1727 displays amplitudes of superhumps that are largest in the earliest stage of the superoutburst; similarly to RZ LMi (Osaki, 1995b), it has an extremely short superoutburst duration.

Besides of MASTER 1727, there are two other known ER UMa-type objects around the period minimum, RZ LMi (Nogami et al., 1995) and DI UMa (Kato et al., 1996). MASTER 1727 is the next after DI UMa object on the standard CV evolutionary path. The evolutionary state of RZ LMi may be different (Kato et al., 2016b).

According to light curve simulations based on the thermal-tidal instability model of SU UMa stars (Osaki, 1995a), the ER UMa-type stars have a high rate of mass transfer, about $\sim 10$ times higher than that expected from the $\mathrm{CV}$ evolution. In our case, for the shortest supercycle of about $50 \mathrm{~d}$, two possibilities correspond to the mass-transfer rate of 4 or $7 \dot{M}$, where $\dot{M}$ is the mass-transfer rate in units of $10^{16} \mathrm{gs}^{-1}$ for the disk radius $0.35 \mathrm{a}$, where a is the binary separation. 


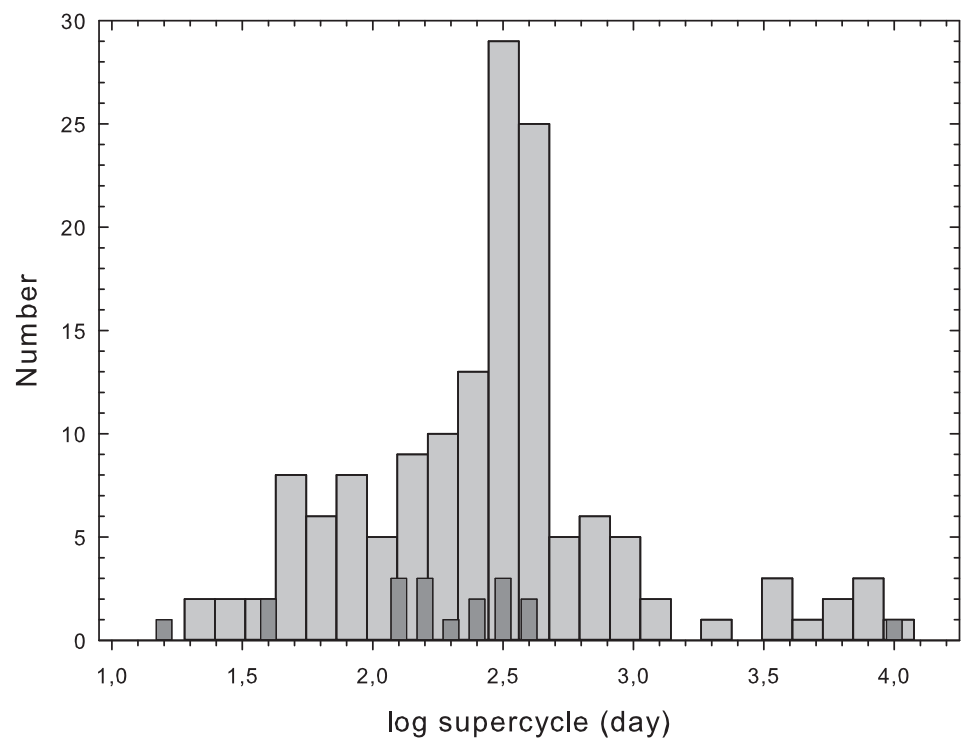

Figure 14. A histogram of supercycles distribution for the SU UMa-type CVs based on the avalable data taken from Ritter \& Kolb (2003), edition 7.24; Pavlenko et al. (2019); Antonyuk \& Pavlenko (2013). Data from Kato et al. (1999) are marked by dark rectangles.

The low mass ratio (and, hence, a small mass of the secondary) is a key parameter that may explain the peculiarities of MASTER 1727. A small mass of the secondary causes the weaker tidal torques compared to the usual SU UMa-type stars (Osaki, 1995b). The weak tidal torques lead to an increase in the radius of the accretion disc at the end of the superoutburst and a reduction in its duration since a smaller angular momentum will be removed during the superoutburst. This could explain a shortening of a supercycle because it takes a shorter time to replenish the angular momentum reservoir in the disc. Osaki (1995b) showed that dependence of a number of normal outbursts on supercycle for ordinary and ER UMa-type DNe (i.e. for DNe with low and high $\dot{M}$ ) is the opposite: while this number is inversely proportional to the supercycle for stars with low and moderate $\dot{M}$, it is proportional to the supercycle for stars with high $\dot{M}$.

A big radius of the disk could provide the possibility of accumulation of cold matter in the outer parts of the accretion disc beyond the 3:1 resonance that may be the cause of rebrightenings. Note that the colour indices behaviour of MASTER 1727 during the superoutburst is also similar to that in WZ Sge-type stars (see e.g. Imada et al. (2018); Neustroev et al. (2017)). A strong variations 
in $\dot{M}$ probably may modify a morphology of the superoutburst from a "clear" superoutburst to a superoutburst with rebrightenings.

The supercycle variation of MASTER 1727 deserves special attention. According to Osaki (1995b), a two-fold increase in the supercycle corresponds to a $\sim$ two-fold decrease of $\dot{M}$. There are several known examples with variable supercycles in active novae where the supercycle changes in a form of secular increase and/or quasi-periodic oscillations as was shown by Kato (2001), Otulakowska-Hypka \& Olech (2014), Zemko et al. (2013). Generally, the nature of these variations is still an open question. However, the tendency of the secular increase in the supercycle, which means a gradual increase in $\dot{M}$, suggests that these objects (at least some of them) could experience a transition between DNe and nova-likes. Thus Kato et al. (2016b) applied this idea for a systematic increase of the supercycle length in RZ LMi.

There are several known postnovae stars of different evolution status (i.e., orbital periods) showing some activity that is probably caused by a high mass transfer rate after the nova explosion. Thus in the renown classical Nova Cygni 1975 (V1500 Cyg), which is an asynchronous polar with the orbital period of $0.14 \mathrm{~d}$, a strong eruption heated the WD up to the $\sim 100000 \mathrm{~K}$ (Schmidt et al., 1995). The hot WD irradiated the secondary and formed a radiationdriven wind (Katz, 1991), which during the first three years after the eruption could have an even stronger decelerating effect on the orbital motion of the WD than the magnetic field. The observational evidence of this was shown by Pavlenko \& Malanushenko (1995). At present (45 years after the WD eruption) the white dwarf is still hot and produces $\sim 1.5$-mag reflection effect of the secondary (Pavlenko et al., 2018).

It was shown by Patterson et al. (2020) that the mass-transfer rate in recurrent novae T Pyx and IM Nor is unnaturally high for their state of evolution (orbital periods are $0.076 \mathrm{~d}$ and $0.103 \mathrm{~d}$, respectively) and supposed that it is caused by a strong and long-lasting irradiation effect of the secondary component powered by a WD eruption.

MASTER 1727, with an orbital period of $0.057 \mathrm{~d}$ and mass ratio 0.08 is a low-q extension of ER UMa stars. The mass-transfer rate should be accordingly higher than what is expected from gravitational radiation only. The only currently known mechanism could be a post-nova state. They suggest that the object underwent a nova eruption relatively recently - hundreds of years ago. (Note that checking the DASCH archive $^{5}$ did not reveal a nova outburst over the past $\sim 100$ years). This object would provide the evidence that a nova eruption can occur even if CVs are near the period minimum (probably the first observational evidence for that).

\footnotetext{
${ }^{5}$ http://dasch.rc.fas.harvard.edu/
} 


\section{Summary}

- The spectrum of MASTER J1727 that was obtained in 2020 quiescence contained $\mathrm{H}$ lines and no He lines;

- The continuous photometry in 2019 over $\sim 160 \mathrm{~d}$ revealed a supercycle length to be $52 \mathrm{~d}$, but the supercycle variability was found from 50 to $100 \mathrm{~d}$ on the $\sim 5$.5-year scale. This implies as high as two times variations in the mass ratio;

- We found a mean period of positive superhumps to be $0.058029 \mathrm{~d}$ during a rather short 7-d superoutburst plateau;

- Late superhumps with a slightly shorter period of $0.057915 \mathrm{~d}$ were detected after the superoutburst. They lasted about $20 \mathrm{~d}$ up to the start of a normal outburst;

- In 2019 we found the orbital period of $0.057026 \mathrm{~d}$ during quiescence after the normal outburst and established its identity with those found earlier by spectroscopy (Thorstensen et al., 2016); the absence of an eclipse in the orbital light curve and its moderate amplitude is consistent with the orbital inclination of about $40^{\circ}$ that we found from spectral observations;

- A blue peak of $V-I c$ and $B-R c$ colour indices coincides with a minimum of the superhump light curve during the superoutburst and those of $B-R c$ colour-index coincides with a rising branch of the late superhumps in quiescence;

- We estimated a low mass ratio of 0.08 and a small mass of the secondary $\sim 0.06 \mathrm{M} \odot$ that could explain most of the observed peculiarities of MASTER J1727 in the frame of thermal-tidal instability of accretion discs;

- The high mass-transfer rate indicates that in the relatively recent history, the MASTER J1727 could undergo a nova eruption. If so, it means that an eruption can occur even if the star is near the period minimum.

Acknowledgements. We are grateful to the anonymous referee for the valuable comments which helped to improve this paper. Elena Pavlenko is grateful to Nikolaus Vogt for the valuable discussion about the MASTER 1727 at the "Compact White Dwarf Binaries" Conference held in Erevan (2019) and Natali Katysheva for careful reading of the manuscript. Observations with the SAO RAS telescopes are supported by the Ministry of Science and Higher Education of the Russian Federation (including agreement No 05.619.21.0016, project ID RFMEFI61919X0016); Observations with CrAO RAS telescopes are supported by the grant of RSF (project number 19-7210063). The work of Shimansky was funded by RFBR, project number 18-42-160003. The work of Maksim Gabdeev was funded by the subsidy 671-2020-0052 allocated to the Kazan Federal University for the state assignment in the sphere of scientific activities and was funded by RFBR, project number 19-32-60021. The work of Dubovský was supported by the Slovak Research and Development Agency under contract No. APVV-15-0458. 


\section{References}

Andronov, I. L. \& Baklanov, A. V., Algorithm of the artificial comparison star for the CCD photometry. 2004, Astronomical School's Report, 5, 264, DOI: 10.18372/24116602.05 .1264

Antonyuk, O. I. \& Pavlenko, E. P., Peculiarities of the SU UMa-Type Dwarf Nova V1504 Cyg Behavior in 1994 - 2012 Years. 2013, Astrophysics, 56, 539, DOI: 10.1007/s10511-013-9306-5

Baraffe, I., Chabrier, G., Barman, T. S., Allard, F., \& Hauschildt, P. H., Evolutionary models for cool brown dwarfs and extrasolar giant planets. The case of HD 209458. 2003, Astronomy and Astrophysics, 402, 701, DOI: 10.1051/0004-6361:20030252

Borisov, N. V., Gabdeev, M. M., Shimansky, V. V., Katysheva, N. A., \& Shugarov, S. Y., Spectral and photometric studies of polar CRTS CSS $130604 \mathrm{~J}$ 215427+155714. 2017, Astrophysical Bulletin, 72, 184, DOI: $10.1134 / \mathrm{S} 1990341317020092$

Cannizzo, J. K. \& Ramsay, G., The Superoutburst Duration versus Orbital Period Relation for AM CVn Stars. 2019, Astronomical Journal, 157, 130, DOI: 10.3847/1538$3881 / \mathrm{ab04ac}$

Denisenko, D., Lipunov, V., Gorbovskoy, E., et al., New Bright CV detected by MASTER. 2014, The Astronomer's Telegram, 5724, 1

Eggleton, P. P., Aproximations to the radii of Roche lobes. 1983, Astrophysical Journal, 268, 368, DOI: $10.1086 / 160960$

Fried, R. E., Kemp, J., Patterson, J., et al., Superhumps in Cataclysmic Binaries. XVI. DI Ursae Majoris. 1999, Publications of the ASP, 111, 1275, DOI: 10.1086/316433

Girardi, L., Bressan, A., Bertelli, G., \& Chiosi, C., Evolutionary tracks and isochrones for low- and intermediate-mass stars: From 0.15 to $7 \mathrm{M}_{\text {sun }}$, and from $\mathrm{Z}=0.0004$ to 0.03. 2000, Astronomy and Astrophysics, Supplement, 141, 371, DOI: 10.1051/aas:2000126

Green, M. J., Marsh, T. R., Steeghs, D. T. H., et al., High-speed photometry of Gaia14aae: an eclipsing AM CVn that challenges formation models. 2018, Monthly Notices of the RAS, 476, 1663, DOI: 10.1093/mnras/sty299

Hellier, C. 2001, Cataclysmic Variable Stars (Springer)

Hirose, M. \& Osaki, Y., Hydrodynamic Simulations of Accretion Disks in Cataclysmic Variables: Superhump Phenomenon in SU UMa Stars. 1990, Publications of the ASJ, 42, 135

Imada, A., Yanagisawa, K., \& Kawai, N., On the colour variations of negative superhumps. 2018, Publications of the ASJ, 70, L4, DOI: 10.1093/pasj/psy068

Kato, T., Changing Supercycle of the ER UMa-Type Star V1159 Ori. 2001, Publications of the ASJ, 53, L17, DOI: 10.1093/pasj/53.4.L17

Kato, T., WZ Sge-type dwarf novae. 2015, Publications of the ASJ, 67, 108, DOI: $10.1093 / \mathrm{pasj} / \mathrm{psv} 077$ 
Kato, T., Hambsch, F.-J., Monard, B., et al., Survey of period variations of superhumps in SU UMa-type dwarf novae. VIII. The eighth year (2015-2016). 2016a, Publications of the ASJ, 68, 65, DOI: $10.1093 / \mathrm{pasj} / \mathrm{psw} 064$

Kato, T., Imada, A., Uemura, M., et al., Survey of Period Variations of Superhumps in SU UMa-Type Dwarf Novae. 2009, Publications of the ASJ, 61, S395, DOI: 10.1093/pasj/61.sp2.S395

Kato, T., Ishioka, R., Isogai, K., et al., RZ Leonis Minoris bridging between ER Ursae Majoris-type dwarf nova and nova-like system. 2016b, Publications of the ASJ, 68, 107, DOI: $10.1093 / \mathrm{pasj} / \mathrm{psw} 101$

Kato, T. \& Kunjaya, C., Discovery of a Peculiar SU UMa-Type Dwarf Nova ER Ursae Majoris. 1995, Publications of the ASJ, 47, 163

Kato, T., Nogami, D., \& Baba, H., DI Ursae Majoris: Discovery of an Extreme ER UMa-Type Dwarf Nova with the Shortest Orbital Period. 1996, Publications of the ASJ, 48, L93, DOI: 10.1093/pasj/48.5.L93

Kato, T., Nogami, D., Baba, H., et al., Observation of ER UMa Stars. 1999, in Disk Instabilities in Close Binary Systems, ed. S. Mineshige \& J. C. Wheeler, 45

Kato, T. \& Osaki, Y., New Method of Estimating Binary's Mass Ratios by Using Superhumps. 2013, Publications of the ASJ, 65, 115, DOI: 10.1093/pasj/65.6.115

Kato, T., Stubbings, R., Nelson, P., et al., The nature of V359 Centauri revealed: New long-period SU UMa-type dwarf nova. 2002, Astronomy and Astrophysics, 395, 541, DOI: 10.1051/0004-6361:20021267

Katz, J. I., V1500 Cygni: A Prediction. 1991, Astrophysical Journal, Letters, 374, L59, DOI: $10.1086 / 186071$

Knigge, C., The donor stars of cataclysmic variables. 2006, Monthly Notices of the $R A S$, 373, 484, DOI: 10.1111/j.1365-2966.2006.11096.x

Knigge, C., Baraffe, I., \& Patterson, J., The Evolution of Cataclysmic Variables as Revealed by Their Donor Stars. 2011, Astrophysical Journal, Supplement, 194, 28, DOI: $10.1088 / 0067-0049 / 194 / 2 / 28$

Lubow, S. H., Simulations of Tidally Driven Eccentric Instabilities with Application to Superhumps. 1991, Astrophysical Journal, 381, 268, DOI: 10.1086/170648

Masci, F. J., Laher, R. R., Rusholme, B., et al., The Zwicky Transient Facility: Data Processing, Products, and Archive. 2019, Publications of the ASP, 131, 018003, DOI: $10.1088 / 1538-3873 /$ aae8ac

Montgomery, M. M., Does an Average White Dwarf Have Enough Mass to Prevent Accretion Disk Tilt? 2010, in American Institute of Physics Conference Series, Vol. 1273, American Institute of Physics Conference Series, ed. K. Werner \& T. Rauch, 358-361

Neustroev, V. V., Marsh, T. R., Zharikov, S. V., et al., The remarkable outburst of the highly evolved post-period-minimum dwarf nova SSS J122221.7-311525? 2017, Monthly Notices of the RAS, 467, 597, DOI: 10.1093/mnras/stx084

Nogami, D., Kato, T., Masuda, S., et al., Photometric Observations of an Extreme ER UMa Star, RZ Leonis Minoris. 1995, Publications of the ASJ, 47, 897 
Nogami, D., Uemura, M., Ishioka, R., et al., In-the-gap SU UMa-type dwarf nova, Var73 Dra with a supercycle of about 60 days. 2003, Astronomy and Astrophysics, 404, 1067, DOI: 10.1051/0004-6361:20030509

Osaki, Y., Irradiation-induced mass-overflow instability as a possible cause of superoutbursts in SU UMa stars. 1985, Astronomy and Astrophysics, 144, 369

Osaki, Y., A model for the superoutburst phenomenon of SU Ursae MAjoris stars. 1989, Publications of the ASJ, 41, 1005

Osaki, Y., A Model for a Peculiar SU Ursae Majoris-Type Dwarf Nova ER Ursae Majoris. 1995a, Publications of the ASJ, 47, L11

Osaki, Y., Why Does RZ Leonis Minoris, an Unusual SU UMa Star, Have Such a Short Supercycle? 1995b, Publications of the ASJ, 47, L25

Osaki, Y., Dwarf-Nova Outbursts. 1996, Publications of the ASP, 108, 39, DOI: 10.1086/133689

Osaki, Y. \& Kato, T., Study of Superoutbursts and Superhumps in SU UMa Stars by the Kepler Light Curves of V344 Lyrae and V1504 Cygni. 2013, Publications of the $A S J$, 65, 95, DOI: 10.1093/pasj/65.5.95

Osaki, Y. \& Kato, T., A further study of superoutbursts and superhumps in SU UMa stars by the Kepler light curves of V1504 Cygni and V344 Lyrae. 2014, Publications of the ASJ, 66, 15, DOI: 10.1093/pasj/pst015

Otulakowska-Hypka, M. \& Olech, A., Increasing Supercycle Lengths of Active SU UMa-type Dwarf Novae. 2014, in Astronomical Society of the Pacific Conference Series, Vol. 490, Stellar Novae: Past and Future Decades, ed. P. A. Woudt \& V. A. R. M. Ribeiro, 385

Paczynski, B., A model of accretion disks in close binaries. 1977, Astrophysical Journal, 216, 822, DOI: $10.1086 / 155526$

Patterson, J., Kemp, J., Jensen, L., et al., Superhumps in Cataclysmic Binaries. XVIII. IY Ursae Majoris. 2000, Publications of the ASP, 112, 1567, DOI: 10.1086/317724

Patterson, J., Kemp, J., Monard, B., et al., IM Normae: The Death Spiral of a Cataclysmic Variable? 2020, arXiv e-prints, arXiv:2010.07812

Pavlenko, E., Antonyuk, K., Pit, N., et al., Evolution of negative superhumps. 2019, in Compact White Dwarf Binaries, ed. G. H. Tovmassian \& B. T. Gansicke, 39

Pavlenko, E. P. \& Malanushenko, V. P., The Peculiarity of V1500 Cyg Syncronization. 1995, in Astrophysics and Space Science Library, Dordrecht Kluwer Academic Publishers, Vol. 205, Cataclysmic Variables, ed. A. Bianchini, M. della Valle, \& M. Orio, 172

Pavlenko, E. P., Mason, P. A., Sosnovskij, A. A., et al., Asynchronous polar V1500 Cyg: orbital, spin, and beat periods. 2018, Monthly Notices of the RAS, 479, 341, DOI: $10.1093 / \mathrm{mnras} / \mathrm{sty} 1494$

Pavlenko, E. P., Sosnovskij, A. A., Antoniuk, K. A., et al., Humps and Superhumps in the SU UMa-Type Dwarf Nova System IRXS J161659.5+620014. 2020, Astrophysics, DOI: $10.1007 / \mathrm{s} 10511-020-09653-0$

Pel't, Y. 1980, Frequency analysis of astronomical time series. (Valgus, Tallin) 
Ritter, H. \& Kolb, U., Catalogue of cataclysmic binaries, low-mass X-ray binaries and related objects (Seventh edition). 2003, Astronomy and Astrophysics, 404, 301, DOI: 10.1051/0004-6361:20030330

Ritter, H. \& Kolb, U., VizieR Online Data Catalog: Cataclysmic Binaries, LMXBs, and related objects (Ritter+, 2004). 2011, VizieR Online Data Catalog, B/cb

Robertson, J. W., Honeycutt, R. K., \& Turner, G. W., RZ Leonis Minoris, PG 0943+521, and V1159 Orionis: Three Cataclysmic Variables with Similar and Unusual Outburst Behavior. 1995, Publications of the ASP, 107, 443, DOI: $10.1086 / 133572$

Schmidt, G. D., Liebert, J., \& Stockman, H. S., Detection of the Hot White Dwarf in the Magnetic Nova V1500 Cygni with the Hubble Space Telescope. 1995, Astrophysical Journal, 441, 414, DOI: 10.1086/175365

Shappee, B. J., Prieto, J. L., Grupe, D., et al., The Man behind the Curtain: XRays Drive the UV through NIR Variability in the 2013 Active Galactic Nucleus Outburst in NGC 2617. 2014, Astrophysical Journal, 788, 48, DOI: 10.1088/0004$637 \mathrm{X} / 788 / 1 / 48$

Smak, J., Statistical analysis of outbursts and superoutbursts of VW Hyi. 1985, Acta Astron., 35, 357

Sosnovskij, A., Pavlenko, E., Pit, N., \& Antoniuk, K., NY Her: possible discovery of negative superhumps. 2017, Information Bulletin on Variable Stars, 6216, 1, DOI: 10.22444/IBVS.6216

Thorstensen, J. R., Alper, E. H., \& Weil, K. E., A Trip to the Cataclysmic Binary Zoo: Detailed Follow-up of 35 Recently Discovered Systems. 2016, Astronomical Journal, 152, 226, DOI: $10.3847 / 1538-3881 / 152 / 6 / 226$

Uemura, M., Kato, T., Ishioka, R., et al., Superhump Evolution in the Ultrashort Period Dwarf Nova 1RXS J232953.9+062814. 2002, Publications of the ASJ, 54, 599, DOI: $10.1093 / \mathrm{pasj} / 54.4 .599$

Vogt, N., VW Hydri revisited : conclusions on dwarf nova outburst models. 1983, Astronomy and Astrophysics, 118, 95

Warner, B., Cataclysmic variable stars. 1995, Cambridge Astrophysics Series, 28

Warner, B. 2003, Cataclysmic Variable Stars (Cambridge University Press)

Whitehurst, R., Numerical simulations of accretion discs - I. Superhumps : a tidal phenomenon of accretion discs. 1988, Monthly Notices of the RAS, 232, 35, DOI: $10.1093 /$ mnras $/ 232.1 .35$

Zemko, P., Kato, T., \& Shugarov, S. Y., Detection of Change in Supercycles in ER Ursae Majoris. 2013, Publications of the ASJ, 65, 54, DOI: 10.1093/pasj/65.3.54 